\title{
Controlled Movement Processing: Superior Colliculus Activity Associated with Countermanded Saccades
}

\author{
Martin Paré ${ }^{1}$ and Doug P. Hanes ${ }^{2}$ \\ ${ }^{1}$ Department of Physiology, Queen's University, Kingston, Ontario, Canada K7L 3N6, and 2Laboratory of Sensorimotor Research, National Eye Institute, \\ National Institutes of Health, Bethesda, Maryland 20892
}

We investigated whether the monkey superior colliculus (SC), an important midbrain structure for the regulation of saccadic eye movements, contains neurons with activity patterns sufficient to control both the cancellation and the production of saccades. We used a countermanding task to manipulate the probability that, after the presentation of a stop signal, the monkeys canceled a saccade that was planned in response to an eccentric visual stimulus. By modeling each animal's behavioral responses, with a race between GO and STOP processes leading up to either saccade initiation or cancellation, we estimated that saccade cancellation took on average 110 msec. Neurons recorded in the superior colliculus intermediate layers during this task exhibited the discharge properties expected from neurons closely involved in behavioral control. Both saccade- and fixation-related discharged differently when saccades were countermanded instead of executed, and the time at which they changed their activity preceded the behavioral estimate of saccade cancellation obtained from the same trials by 10 and $13 \mathrm{msec}$, respectively. Furthermore, these intervals exceed the minimal amount of time needed for SC activity to influence eye movements. The additional observation that saccade-related neurons discharged significantly less when saccades were countermanded instead of executed suggests that saccades are triggered when these neurons reach a critical activation level. Altogether, these findings provide solid evidence that the superior colliculus contains the necessary neural signals to be directly involved in the decision process that regulates whether a saccade is to be produced.

Key words: superior colliculus; saccade; fixation; motor control; countermanding; monkey

\section{Introduction}

The cognitive control of actions entails a decision process, the outcome of which is either the execution of a voluntary act or its withholding. The ability to withhold a voluntary act is therefore a fundamental demonstration of motor control, and studying the process underlying the inhibition of action can provide valuable insights about executive interventions.

Stopping behavior has been successfully examined with the countermanding paradigm (for review, see Logan, 1994). This paradigm consists of a task that manipulates a subject's ability to withhold responses to a go stimulus when a stop signal is presented occasionally; stopping is easy when a short delay separates the stop signal from the go stimulus and increasingly difficult as the delay increases. The duration required to stop the movements can be estimated reliably by modeling the stopping behavior with a race between a GO process initiated by the go stimulus and a STOP process initiated by the stop signal. The behavioral outcome is determined by whichever process first crosses a finish line (Logan and Cowan, 1984). However, this model provides only

Received 0ct. 16, 2002; revised May 6, 2003; accepted May 7, 2003.

This work was supported by the National Eye Institute and the Canadian Institutes of Health Research (M.P.). M.P. holds a New Investigator Award from the Canadian Institutes of Health Research. We are grateful to Robert H. Wurtz (National Eye Institute) for his extensive support and advice, without which this study would not have been possible. We also thank R. Pinkerton and L. Ekstrom for their contributions to the data analysis as well as N. Bains for her advice on statistics.

Correspondence should be addressed to Dr. Martin Paré, Queen's University, Department of Physiology, Botterell Hall, Room 438, Kingston, Ontario, Canada K7L 3N6. E-mail: pare@biomed.queensu.ca.

Copyright (C) 2003 Society for Neuroscience $\quad$ 0270-6474/03/236480-10\$15.00/0 limited insights into the underlying neural mechanisms by suggesting that movement processing is interruptible only during an initial controlled phase before it reaches a trigger threshold, and a ballistic phase inexorably leads to its completion (Osman et al., 1986; DeJong et al., 1990).

One of our best models of movement regulation has been developed from the study of saccades, the rapid eye movements we make to realign our visual axis. Recently, the countermanding paradigm was adapted to the behavioral and neurophysiological study of the saccadic system (Hanes and Schall, 1995; Hanes et al., 1998; Hanes and Carpenter, 1999; Stuphorn et al., 2000). These neurophysiological studies have concentrated their investigations on prefrontal executive areas and revealed that at least the frontal eye field (FEF) contains neurons with activity patterns sufficient to control saccade cancellation. We wished to continue this fruitful approach in the brainstem to evaluate the contribution of the superior colliculus (SC), perhaps the most important brain structure regulating saccade production.

Several pieces of evidence already suggest that the SC plays an important role in determining whether and when a saccade will be produced. First, the production and latency of saccades are greatly affected after either reversible or irreversible lesions of the SC (Schiller et al., 1980, 1987; Hikosaka and Wurtz, 1985, 1986; Sparks et al., 1990; Aizawa and Wurtz, 1998; Quaia et al., 1998; Hanes and Wurtz, 2001). Second, the latency of saccades evoked by SC stimulation varies with the intensity of the stimulation (Robinson, 1972; Paré et al., 1994; Stanford et al., 1996). Third, 
the level of low-frequency activity displayed in advance of saccades by some SC neurons predicts the reaction times of saccades (Dorris et al., 1997). Last, other SC neurons discharge highfrequency bursts of activity that are coupled with both the occurrence and onset of saccades (Sparks, 1978). Thus, some aspects of the SC activity appear necessary for a saccade to be produced, but it remains unclear whether SC neurons change their activity appropriately when a saccade is countermanded instead of executed. This study used the countermanding paradigm to determine whether the changes in SC neuronal activity patterns are physiologically sufficient (in magnitude and timing) to regulate whether a saccade is to be made. These data have been reported in preliminary form previously (Hanes and Paré, 1998).

\section{Materials and Methods}

Subjects and physiological procedure. Data were collected from two male rhesus monkeys (Macaca mulatta; 7-10 kg) that were cared for in accordance with the Institute of Animal Care and Use Committee and the Public Health Service Policy on the human care and use of laboratory animals. All surgical procedures and electrophysiological techniques have been described previously (Paré and Wurtz, 2001).

Stimuli and apparatus. Experiments were controlled by a 486 personal computer running the UNIX-based real-time experimentation software (Hays et al., 1982), which recorded, at a sample rate of $1 \mathrm{kHz}$, both the isolated action potentials and the monkeys' gaze position that was monitored by the magnetic search coil technique (Fuchs and Robinson, 1966). Head-restrained monkeys were seated in an adjustable primate chair and faced a tangent screen located $57 \mathrm{~cm}$ away, onto which the fixation spot and the saccade targets were rear projected by a laser and a video projector $(60 \mathrm{~Hz}$; noninterlaced $)$, respectively. The projector was synchronized to the computer by the vertical retrace, and the location of visual stimuli on the tangent screen within a vertical retrace cycle was taken into account. The background was uniform gray with a luminance $<0.1 \mathrm{~cd} / \mathrm{m}^{2}$. The fixation spot and the saccade targets subtended $0.25^{\circ}$ of visual angle, and their luminance was $2 \mathrm{~cd} / \mathrm{m}^{2}$.

Behavioral tasks. Neurons were first characterized while the monkeys performed several behavioral tasks, including the memory-guided saccade task and the gap saccade task. All behavioral trials were initiated by the appearance of a central fixation spot, which the monkey was required to fixate within $1000 \mathrm{msec}$ and for a variable $(500-800 \mathrm{msec})$ interval. In the memory-guided saccade task, a peripheral target was briefly presented (100 msec flash), and the fixation spot remained illuminated for an additional 500-1000 msec before disappearing to signal the monkey to make a saccade to the remembered location of the saccade target within $500 \mathrm{msec}$ and then maintain fixation on it for $200-400 \mathrm{msec}$ to correctly perform the task and be rewarded. In the gap saccade task, the fixation spot was extinguished after the initial fixation interval, and the monkey had to maintain steady fixation during a $200 \mathrm{msec}$ gap interval before the saccade target appeared. In all tasks, the saccade target was presented with equal probability either in the center of the response field of the neuron or at the same eccentricity but in the diametrically opposite direction. We also used a fixation-blink task, in which the monkeys had to maintain fixation while the central fixation spot was blinked for a $400-600$ msec interval.

The main data of this study were collected while the monkeys performed the saccade-countermanding task (Fig. 1), which has been described previously (Hanes and Schall, 1995; Hanes et al., 1998). After the initial fixation interval, the central fixation spot disappeared simultaneously with the appearance of a saccade target. For $33 \%$ of the trials, the fixation spot reappeared after a delay, referred to as the stop-signal delay (SSD), and instructed the monkey to inhibit movement production. In those trials in which the stop signal was not presented (CONTROL trials), monkeys were given a liquid reward for generating a single saccade to the peripheral target within $500 \mathrm{msec}$ and by maintaining fixation on the target for $200-400 \mathrm{msec}$. In those trials in which the stop signal was presented (STOP trials), monkeys were rewarded for successfully maintaining fixation for $600 \mathrm{msec}$ after the stop-signal appearance (canceled
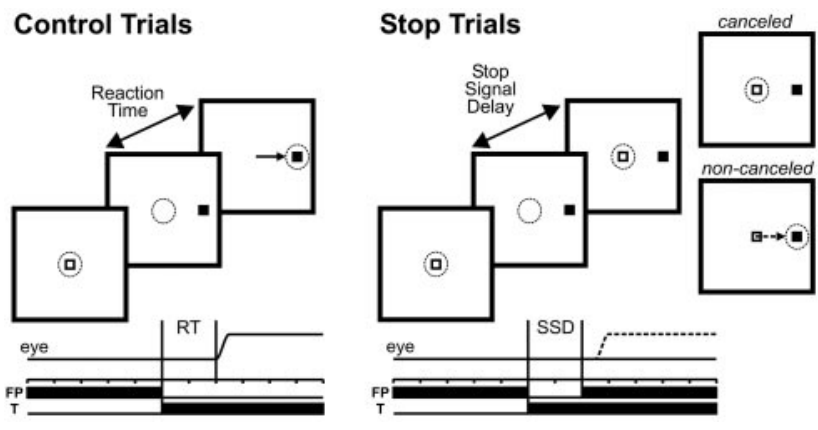

Figure 1. Trial displays for the countermanding saccade task. Each trial began with the fixation of a central fixation point (FP) for a variable interval after which it disappeared and a target (T) simultaneously appeared, either in the response field of the neuron or in the opposite hemifield. In the CONTROL trials, monkeys were rewarded for responding with a single targeting saccade (left). On a fraction of interleaved trials (right), the fixation point reappeared after a variable delay (SSD) and acted as a stop signal instructing the monkeys to withhold saccade initiation. In these STOP trials, they were rewarded for countermanding the planned movement and maintaining fixation on the fixation point (canceled STOP trials). No reward was delivered if they responded with a targeting saccade (noncanceled STOP trials). The dotted circle and arrow indicate current gaze position and saccade vector during each interval, respectively.

trials), but not if they generated a saccade to the peripheral target (noncanceled trials).

Stop-signal delays ranged from a minimum of 25 to a maximum of 230 $\mathrm{msec}$, and we selected for each experimental sessions four different stopsignal delays spaced at a fixed interval (either 40, 50, or $60 \mathrm{msec}$ ). Stopsignal delays were varied according to the monkeys' performance so that at the shortest stop-signal delay, monkeys generally inhibited the movement in $>85 \%$ of the stop-signal trials and at the longest delay, monkeys inhibited the movement in fewer than $15 \%$ of the stop signal trials. The four stop-signal delays were kept constant while recording from an individual neuron. By having a small proportion of STOP trials and limiting the maximum saccade reaction time to $500 \mathrm{msec}$ in CONTROL trials, we ensured that the monkeys produced a speeded response to the target presentation and did not adopt a strategy of postponing their response until they could determine whether a stop signal was to be presented.

Neuronal classification. We collected sufficient data in the necessary countermanding trial conditions from a total of 48 SC neurons that exhibited task-related activity in the countermanding saccade task. Neurons with saccade-related activity were defined as neurons that exhibited an increased discharge rate before memory-guided saccades. These neurons could additionally exhibit a phasic response to the target presentation, a delay-period activity, or both. Using previously established criteria (Munoz and Wurtz, 1995), we subdivided this sample of neurons into two classes: buildup and burst neurons. The analysis results for the two classes of neurons did not differ, and we therefore will refer to these neurons simply as saccade-related neurons. A total of 32 saccade-related neurons were included in this study.

We also recorded from neurons within the rostro-lateral portion of the SC that displayed fixation-related activity, which we defined as providing an extraretinal fixation signal. The rationale for this has been exposed previously (Munoz and Wurtz, 1993; Dorris and Munoz, 1995). In the gap saccade task, these fixation-related neurons increased their discharge rate after fixation of the central fixation spot and a pause in activity before and during saccades. Their rate of discharge was maintained, albeit at a lower rate, when the fixation spot was momentarily removed and the monkey maintained the same gaze angle during both the gap period of the gap saccade task and the blink period of the fixation-blink task. A total of 10 fixation-related neurons were recorded in this study. Six neurons with neither significant saccade activity during the memory-guided saccade task nor fixation activity were excluded from the analysis.

Data analysis. During off-line analyses, saccades were detected and marked using a computer program that identified the beginning and end of each saccade using velocity and acceleration threshold criteria and template correlation as described by Waitzman et al. (1991). An experi- 
menter verified these events to ensure accuracy. Reaction time was measured as the interval from target appearance to the beginning of the saccade.

To visualize the neural data, rasters of neuronal discharge and continuously varying spike density functions were aligned on the time of the saccade target presentation. Spike density functions were constructed by convolving spike trains with a combination of growth and decay exponential functions that resembled a postsynaptic potential: $R(t)=[1-$ $\left.\exp \left(-t / \tau_{g}\right)\right] \cdot\left[\exp \left(-t / \tau_{d}\right)\right]$, where rate as a function of time $[R(t)]$ varies according to $\tau_{g}$ (the time constant for the growth phase) and $\tau_{d}$ (the time constant for the decay phase). We used values for $\tau_{g}$ and $\tau_{d}$ (1 and 20 msec) (Hanes and Schall, 1996) that had been estimated from physiological studies of excitatory synapses (Sayer et al., 1990; Mason et al., 1991). Note that only spikes that occurred before saccade initiation were used in the computation of the spike density functions (Hanes et al., 1998).

Inhibition functions were constructed that plotted the probability of noncanceled trials as a function of stop-signal delay. To derive reliable parameter estimates, the inhibition data points were fit with a cumulative Weibull function of the form: $W(t)=\gamma-(\gamma-\delta) \cdot \exp \left[-(t / \alpha)^{\beta}\right]$, where $t$ is the time after target presentation, $\alpha$ is the time at which the inhibition function reaches $64 \%$ of its full growth, $\beta$ is the slope, $\gamma$ is the maximum value of the inhibition function, and $\delta$ is the minimum value of the inhibition function. The value of $\gamma$ generally approached 1 (mean, 0.93 ), whereas $\delta$ was usually close to 0 (mean, 0.04 ). All of the Weibull function fits had correlation coefficients $>0.83$ (mean, 0.98 ).

To quantify the time course of the neuronal activation during STOP and CONTROL trials, we calculated a differential spike density function by subtracting the average spike density function associated with each type of trial. The time at which significant differential activation began was defined as the instant when the differential spike density function exceeded by two SD the mean difference in baseline activity, provided the difference remained above this threshold for $50 \mathrm{msec}$. We sampled the baseline activity during either a $600 \mathrm{msec}$ interval before target onset (if the neuron displayed background activity) or a variable interval from the activation onset of the neuron until the earliest possible change in activation, which we estimated from our sample to be $40 \mathrm{msec}$ before the behavioral estimate of saccade cancellation.

\section{Results}

\section{Behavioral estimate of saccade cancellation}

The first goal of this study consisted of obtaining a behavioral estimate of the length of time needed to cancel a saccade. This duration, also known as the stop signal reaction time (SSRT), is a quantity that is not directly observable. However, it can be indirectly estimated from the behavioral performance in the countermanding task, which is captured by two quantifications. From the CONTROL trials, the distribution of reaction times can be constructed to inform us about the overt process of movement production (Fig. 2C). From the STOP trials, the covert process of movement cancellation is reflected in the inhibition function (i.e., the probability of not canceling a saccade plotted as a function of the stop-signal delays) (Fig. $2 \mathrm{~A}$ ). In these experiments, as in previous reports, monkeys successfully withheld their saccades with very short stop-signal delays, but they increasingly failed to do so as the stop-signal delay was lengthened.

This STOP trial behavior can be explained and the duration of the covert inhibitory process can be estimated by a race model (Fig. $2 \mathrm{~B}$ ) between two independent processes: (1) a GO process that prepares and initiates the movement in response to the presentation of the target, and (2) a STOP process initiated by the STOP signal that can inhibit the planned movement. The two processes race with variable rise time toward a finish line, and which ever wins the race dictates the ensuing behavior. If the GO process ends first, the saccade will proceed without hindrance (noncanceled trial). If the STOP process ends first, the saccade will not be produced (canceled trial). When the stop signal is not
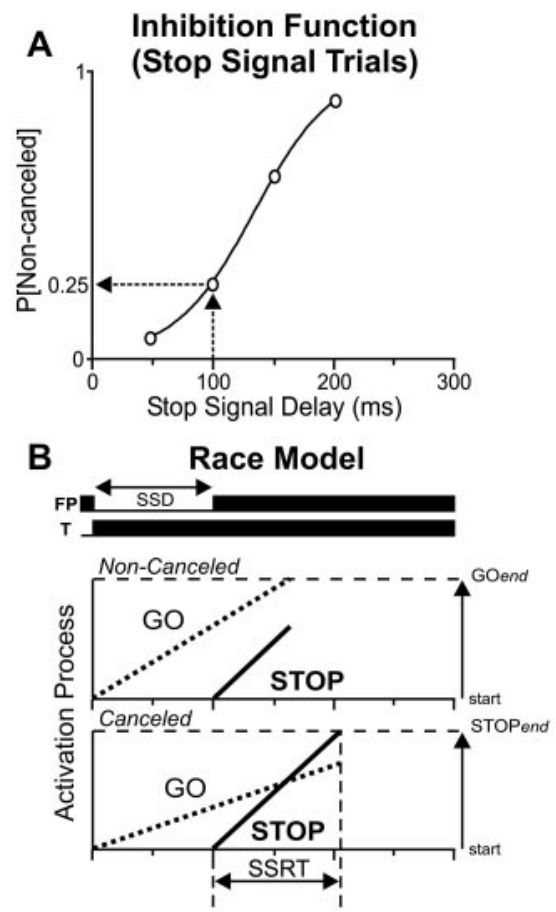

\section{Reaction Time Distribution (Control Trials)}

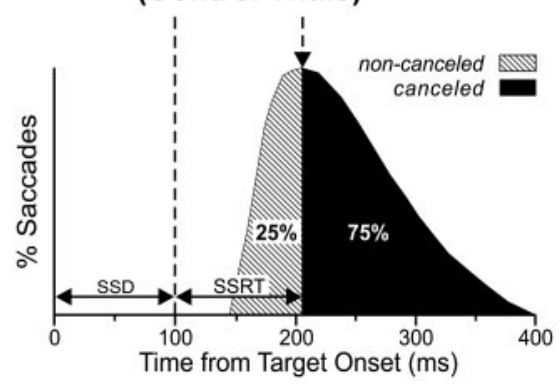

Figure 2. Estimation of the SSRT with the integration method. A, Data from the STOP trials yield an inhibition function, the probability that the monkey failed to countermand the targeting saccade (noncanceled STOP trials) as a function of stop-signal delay. $B$, The race model consists of a GO process (dotted line) and a STOP process (solid line) racing independently toward their respective threshold (broken horizontal line). The $G 0$ and STOP processes are initiated by the presentation of the saccade target and the stop signal, respectively. In STOP trials, the STOP process begins after the $G 0$ process has begun. If the $G 0$ process finishes first, then the saccade will not be canceled. In contrast, if the STOP process finishes before the GO process, then the saccade is canceled. The duration of this stop process is the SSRT. C, The SSRT at each SSD can be determined by integrating the distribution of the reaction times of the saccades made in the CONTROL trials, beginning at zero, until the integral equals the probability of noncanceled trials observed at each SSD. The time value at that point indicates the time that the STOP process ended. Thus, the interval from the presentation of the stop signal to this time value represents the SSRT.

presented, as in the CONTROL trials, the GO process is exclusively active, and the distribution of finish times of this process is represented in the distribution of the saccade reaction times. Although it is rather easy to estimate the GO process, the STOP process can, however, only be approximated with the help of the race model. To estimate the SSRT from each experimental session $(n=48)$ that was performed by the two monkeys and while each neuron was recorded, we used two estimation methods derived from this race model. This approach has been described in detail previously (Logan and Cowan, 1984; Hanes et al., 1998), and it is closely related to analyses performed previously on data from double-step saccade tasks (Lisberger et al., 1975; Becker and Jürgens, 1979). 
Table 1. SSRT estimates (mean \pm SE; in milliseconds) for the two monkeys

\begin{tabular}{llll}
\hline & \multicolumn{2}{l}{ Estimation method } & \\
\cline { 2 - 4 } & Integration & Mean & Average \\
\hline Monkey B $(n=25)$ & $105 \pm 2$ & $104 \pm 3$ & $105 \pm 2$ \\
Monkey M $(n=23)$ & $110 \pm 2$ & $122 \pm 4$ & $116 \pm 2$ \\
Both $(n=48)$ & $108 \pm 1$ & $113 \pm 3$ & $110 \pm 2$ \\
\hline
\end{tabular}

The first estimation method provides an estimate of the SSRT at each stop-signal delay by assuming that the SSRT is a constant (Fig. 2C). Although this assumption seems implausible, it is not crucial because its violation has been demonstrated to not change the estimation substantially (Logan and Cowan, 1984; DeJong et al., 1990; Band et al., 2003). This method estimates, for a given stop-signal delay, the point in time at which the STOP process finishes by integrating the observed distribution of saccade reaction times in the CONTROL trials, beginning at the time of target presentation until the integral equals the observed proportion of noncanceled trials when given a stop signal after that stop-signal delay. This point limit of the integral corresponds to the saccades with the longest reaction time possible before they all become inhibited by the STOP process. Thus, the interval from the appearance of the stop signal and this finish line represents the SSRT at this stopsignal delay. Like others, we observed that the SSRTs calculated with this method varied somewhat with the stop-signal delays, and we therefore averaged their values across the four different delays. With this estimation method, the SSRT averaged $108 \mathrm{msec}$ across both monkeys and all recording sessions (Table 1).

The second estimation method assumes that the SSRT is a random variable and provides an estimate of its mean. Logan and Cowan (1984) showed mathematically that the mean SSRT is equal to the difference between the mean reaction time during CONTROL trials and the mean value of the inhibition function obtained from the STOP trials. We determined the mean of each inhibition function by treating it as a cumulative distribution and converting it into a probability density function. We first fitted each set of inhibition data points with a Weibull function, $W(t)$ (see Materials and Methods). The mean of the function was then calculated as the difference between the probability of not canceling a saccade at a given stop-signal delay $(t)$ and the probability at the preceding stop-signal delay $(t-1)$ multiplied by the given stop-signal delay summed over all stop-signal delays (Logan and Cowan, 1984). To account for the fact that most inhibition functions had a minimum of $>0$ and a maximum of $<1$, or both, we rescaled the mean of the inhibition function by dividing the mean of the inhibition function by the difference between the maximum and minimum probabilities of noncanceled saccades (Hanes et al., 1998). Thus, the estimate of the mean of the best-fit inhibition function is: $\Sigma[W(t)-W(t-1)] \cdot t /\left[W\left(t_{\text {max }}\right)-W\left(t_{\text {min }}\right)\right]$, where $t$ ranges from the minimum to the maximum stop-signal delay in $1 \mathrm{msec}$ intervals. With this estimation method, the SSRT averaged $113 \mathrm{msec}$ (Table 1), a value not significantly different from that obtained with the integration method (paired $t$ test; $t=1.54 ; \mathrm{df}=47 ; p=0.13$ ).

In theory, the two estimation methods are equally valid (Logan and Cowan, 1984). We therefore obtained an overall behavioral estimate of saccade cancellation for each recording session by averaging the SSRT derived from both estimation methods. This estimated SSRT averaged $110 \mathrm{msec}$ and its distribution was unimodal, spanning $<50 \mathrm{msec}$ (range, 90 to 134). In summary, our behavioral analysis indicates that once the stop signal appeared, it took $110 \mathrm{msec}$ for the monkeys to cancel the saccadic program triggered by the visual target presentation.

\section{Neural estimate of saccade cancellation}

The second goal of this study was to obtain a neural estimate of saccade cancellation from the SC neurons recorded while the monkeys performed the countermanding task. To do so, we compared the neuronal activity associated with the STOP trials, during which the saccades were canceled, with the neuronal activity exhibited during CONTROL trials. However, not all CONTROL trials were included in this analysis. In the canceled STOP trials, the saccade production was inhibited, because the STOP process finished before the GO process. Thus, the only valid CONTROL trials that can be compared with the canceled STOP trials are those in which the saccade initiation would have been canceled if the stop signal had been presented (Hanes et al., 1998). These are the trials in which the GO process was slow enough that the STOP process would have finished before the GO process. This subset of corresponding CONTROL trials, identified by the dark region of the example distribution of saccade reaction times in Figure $2 C$, corresponds to the saccades that had reaction times exceeding the stop-signal delay plus the SSRT calculated from the same data. With this valid comparison, the neural estimate of saccade cancellation can be defined as the time when the activity in these two sets of trials becomes significantly different.

To influence behavior, a saccade-related neuron must discharge differently (i.e., more) during corresponding CONTROL trials than canceled STOP trials. Furthermore, for the neuron to be directly involved in canceling the saccade, the differential activity must occur early enough before the behavioral estimate of saccade cancellation. Figure 3 shows the activity of one representative saccade-related neuron recorded during the countermanding task. Figure 3, $A$ and $B$, shows the stimulus-aligned activity during canceled STOP trials and corresponding CONTROL trials, respectively. In the CONTROL trials, the activation of the neuron began after some delay relative to the stimulus onset and gradually grew toward a saccade-related burst of action potentials. In the STOP trials, when the saccades were successfully canceled, a similar initial activation was abruptly truncated some time after the STOP signal. In Figure $3 C$, the spike density functions computed for the activity associated with these two types of trials are superimposed along with the differential spike density function and the time at which it reaches a significance level (see Materials and Methods). This time marks the neural estimate of saccade cancellation and can be contrasted with the SSRT obtained from these trials $(94 \mathrm{msec})$. In the example neuron, the former preceded the latter by $9 \mathrm{msec}$. Furthermore, the discharge rate of the neuron, as measured in a $40 \mathrm{msec}$ interval centered on the behavioral estimate of saccade cancellation, was significantly greater in CONTROL trials $(214 \mathrm{~Hz})$ than in canceled STOP trials $(88 \mathrm{~Hz} ; t=5.95 ; \mathrm{df}=87 ; p<0.0001)$. Thus, the activity of this neuron changed significantly when saccades were canceled as well as in advance of the behavioral estimate of cancellation, thereby suggesting that it has activity sufficient to be directly involved in canceling the planned saccade.

We calculated the discharge rate of each SC neuron during a $40 \mathrm{msec}$ interval centered on the behavioral estimate of saccade cancellation for both the canceled STOP trials and their corresponding CONTROL trials. Figure $4 A$ shows the distribution of the ratios between these discharge rates calculated at each stopsignal delay (with at least five trials) for each saccade-related neuron. All but one $(97 \% ; 31$ of 32$)$ of the saccade-related neurons had a significant activity ratio in at least one stop-signal delay $(t$ test; $p<0.01$ ), and 95\% (88 of 93) of the ratios from all of the stop-signal delays available from all saccade-related neurons were significantly $>1$. Overall, the ratios ranged from 0.89 to 93.06, 
with a mean $( \pm S E)$ of $7.86 \pm 1.30$. Thus, nearly all saccade-related neurons displayed a discharge rate around the time of saccade cancellation that was significantly less in canceled STOP trials than in their corresponding CONTROL trials.

We also determined the relative timing between the estimate of saccade cancellation and the behavioral estimate of saccade cancellation of each neuron obtained from the analysis of the behavioral data collected during the same experimental session (Fig. $4 B$ ). It was possible to calculate a neural estimate of saccade cancellation in at least one stop-signal delay for each of the saccade-related neurons, and this estimate occurred before the behavioral estimate in 94\% (30 of 32) of the neurons. These neural estimates preceded the behavioral estimates, on average, by $10 \mathrm{msec}(\mathrm{SE}=1$; range, -36 to $24 ; n=92$ ). This time difference approximates the SC minimal efferent delay, which has been estimated to be $8 \mathrm{msec}$ (Miyashita and Hikosaka, 1996). Also, $88 \%$ (28 of 32 ) of the neurons significantly changed their activity at least 8 msec before the time of the behavioral estimate of saccade cancellation in at least one stop-signal delay. The fact that the change in activity of nearly all SC neurons preceded the change in behavior within the SC minimal efferent delay demonstrates that SC neuronal activity is sufficient to cancel saccade production.

\section{Maximum activation during the controlled phase of movement processing}

The countermanding task allows us to observe the neural signals during the controlled phase of movement processing. As seen in Figure $3 A$, the activation of saccade-related neurons could be dissociated from saccade production during STOP trials when the saccades were successfully canceled, but it often reached a substantial level. We quantified that level of activity by measuring, for each neuron, the peak of the stimulus-aligned spike density function associated with canceled STOP trials at each stopsignal delay, and we contrasted this value with that of the peak of the saccade-aligned spike density function associated with all CONTROL trials (Fig. 5). This comparison is particularly important to test the hypothesis that the SC neuronal activation must reach a critical threshold to trigger a saccade, in which case the level of activity observed in canceled STOP trials should at least not exceed the maximum activation level observed during CONTROL trials. The highest peak activation during canceled STOP trials averaged $109 \mathrm{~Hz}(\mathrm{SE}=8$; range, 51 to 239), whereas the peak activation observed in CONTROL trials averaged $325 \mathrm{~Hz}$ $(\mathrm{SE}=27$; range, 68 to 664 ). A ratio was calculated for each neuron by dividing the highest peak activation during STOP trials by the peak activation during CONTROL trials. This ratio was found to be always $<1$ (range, 0.15 to 0.97 ) and to average 0.4 , which was significantly $<1$ ( $t$ test; $p<0.0001$ ). Thus, although each neuron discharged substantially in STOP trials when the monkey withheld a saccade, its maximum activation level in these trials was always less than in CONTROL trials when the monkey executed a saccade. This result provides additional evidence for the concept of trigger threshold in movement initiation models (Carpenter and Williams, 1995). Evidence in support of the corollary of this concept, that neuronal activity grows toward this threshold, has also been presented (Hanes and Schall, 1996; Paré, 2002).

\section{Independence of GO and STOP processes}

The race model that we used to estimate the SSRT assumes that the GO and STOP processes are stochastically independent. If the two processes interacted, the SSRT would systematically vary with the stop-signal delay, and the timing difference between the neural estimate and the estimated mean SSRT would be erroneous. We conducted a series of behavioral and neural analyses to test whether the independence assumption of the model was respected in our experiments.

If the growth of the STOP process affected the growth of the GO process, one might expect saccades made in the presence of the stop signal (noncanceled STOP trials) to reach reduced eccentricity and peak velocity, as compared with those made in the 

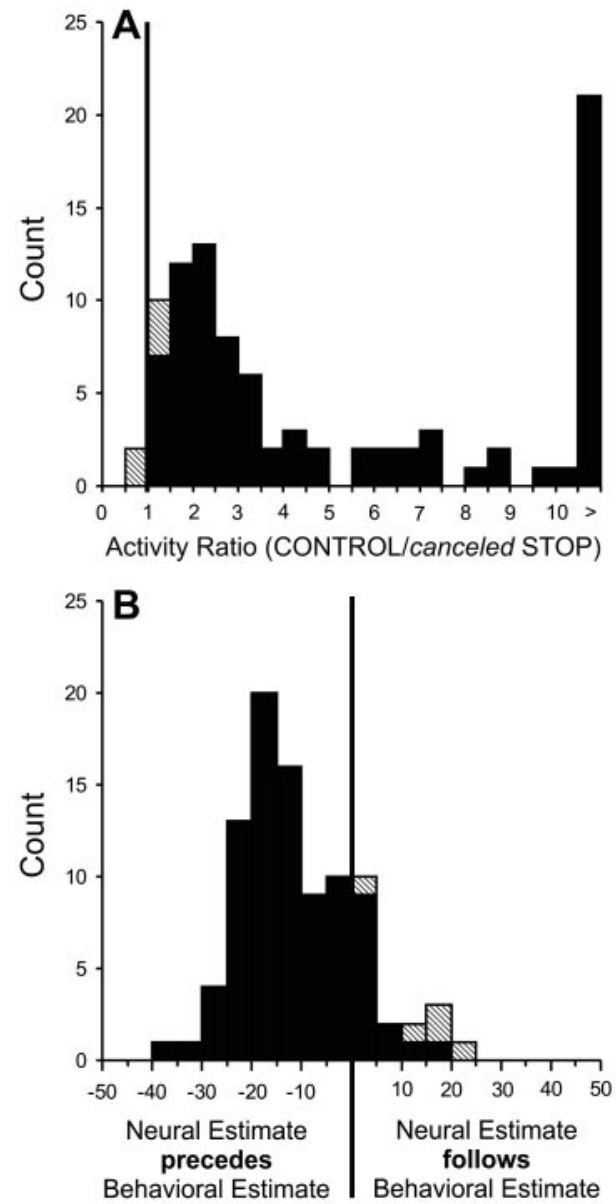

Figure 4. A, Distribution of the ratios of activity in the 40 msec interval centered on the time of the behavioral estimate of saccade cancellation in canceled STOP trials and corresponding CONTROL trials for the group of trials collected in each stop-signal delay in 32SC saccade-related neurons. Solid cells indicate the groups with activity ratios that were significantly greater than unity. $B$, Distribution of the timing difference between the neural and behavioral estimates of saccade cancellation for the same saccade-related neurons. Each stop-signal delay from each neuron contributed one data point.

absence of a stop signal (CONTROL trials) (Hanes and Schall, 1995). We tested this possibility for each group of saccades made to each target in each session, provided that each group had at least 10 movements. The eccentricity of saccades made in noncanceled STOP trials were found to be $1.3 \%$ (mean difference, $0.2^{\circ}$ ) less than that of CONTROL trial saccades; this difference was significant in $27 \%$ ( 22 of 82 ) of comparisons ( $t$ test; $p<0.01$ ). The peak velocity of the saccades made in noncanceled STOP trials were found to be $0.7 \%$ (mean difference, 4 degrees per sec) less than that of CONTROL trial saccades; this difference was significant in only $4 \%$ (3 of 82 ) of comparisons.

An additional behavioral test of the independence assumption consists of determining how well the race model predicts the reaction times of the saccades made in noncanceled STOP trials (Logan and Cowan, 1984; Hanes and Carpenter, 1999). In noncanceled STOP trials, saccades were produced because the GO process finished before the STOP process. Thus, the valid CONTROL trials that can be used to predict the saccade reaction times in these noncanceled STOP trials are those in which the saccade initiation would have been initiated, even if the stop signal had been presented. These are the trials in which the GO process was fast enough that it would have finished before the STOP process if

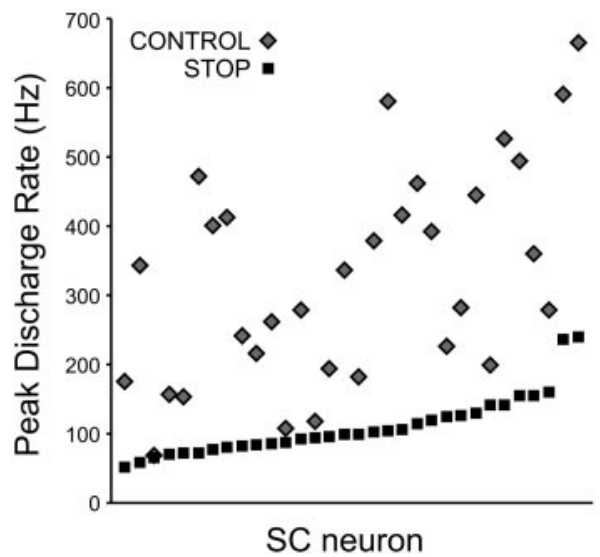

Figure 5. Comparison between the greatest maximum peak activation during canceled STOP trials and the maximum saccade-aligned activation during all CONTROL trials for each of the 32 SC saccade-related neurons.

a stop signal had been presented. This subset of CONTROL trials, identified by the light region of the example distribution of saccade reaction times in Figure $2 C$, corresponds to the ensemble of saccades that had reaction times that were less than the stopsignal delay plus the estimated SSRT. We found that the mean saccade reaction time in these CONTROL trials exceeded the mean saccade reaction time in noncanceled STOP trials by $1.4 \%$ (mean difference, $3 \mathrm{msec}$ ); this difference was significant in only $9 \%$ (10 of 108) of comparisons. In addition, no significant differences were found in the data sets with $>30$ trials $(n=55)$.

We conclude from these behavioral analyses that there is no large violation of the independence assumption of the race model for overt variables related to the outcome of the race between GO and STOP processes. To evaluate the independence of GO and STOP processes as they raced toward the finish line, we compared the neuronal activity associated with the noncanceled STOP trials with that exhibited during CONTROL trials (DeJong et al., 1990; Hanes et al., 1998). If the STOP process interfered with the GO process, the growth of neuronal activity before saccades in noncanceled STOP trials should be slower than that observed before saccades in CONTROL trials. To be valid, the analysis included only STOP trials in which both the GO and STOP processes were active [i.e., trials with saccade reaction times exceeded the stop-

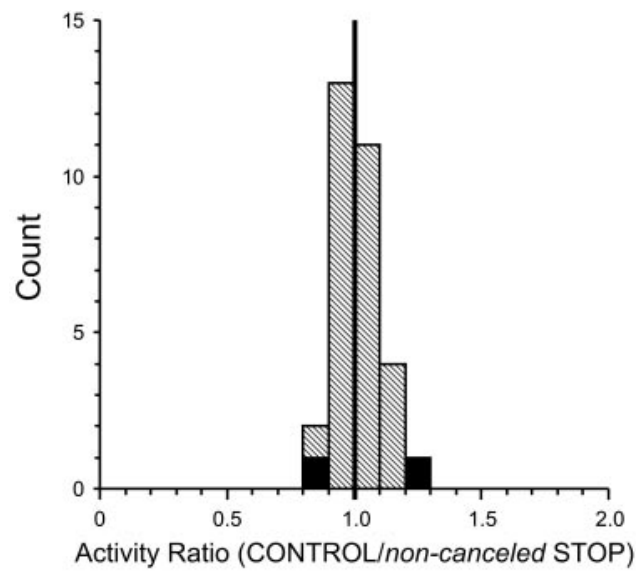

Figure 6. Distribution of the ratios of activity of $\mathrm{SC}$ saccade-related neurons in the $40 \mathrm{msec}$ interval before saccade initiation in noncanceled STOP trials and corresponding CONTROL trials. Each stop-signal delay from each neuron contributed one data point. Solid cells indicate ratios of groups with statistically significant differences. 
signal delay plus the time needed for the SC to register the stop signal presentation (its afferent delay or minimal visual response latency)], which we estimated to be 50 msec (Edelman and Keller, 1996). To provide a valid comparison, this minimum saccade latency restriction was also applied to the corresponding CONTROL trials. Only a significantly greater rate in CONTROL trials could be regarded as evidence against the independence of the GO and STOP processes.

Figure 3, $D$ and $E$, shows the activity of a representative saccade-related neuron during the selected noncanceled STOP trials and corresponding CONTROL trials, respectively. During both types of trials, the activity of this neuron similarly grew and peaked around the time of saccade initiation. The differential spike density function calculated from these noncanceled STOP trials and corresponding CONTROL trials never became significant (Fig. $3 F)$. The average discharge rate of this neuron, as measured in a 40 msec interval ending at the time of saccade initiation, was $216 \mathrm{~Hz}$ during CONTROL trials and $229 \mathrm{~Hz}$ during noncanceled STOP trials, a difference that was not statistically significant $(t=-0.66 ; \mathrm{df}=66 ; p=0.51)$.

A ratio of the discharge rate during noncanceled STOP trials and corresponding CONTROL trials was determined for each stop-signal delay in which sufficient trials were collected with each saccaderelated neuron (Fig. 6). Ninety-four percent (29 of 31 ) of the ratios from all stop-signal delays available from all saccade-related neurons were not significantly different from 1 . Overall, the ratios ranged from 0.83 to 1.21 , with a mean $( \pm S E)$ of $1.01 \pm 0.02$, which was not significantly different from 1 ( $t$ test; $p=0.40$ ). For all of the neurons, the difference between the average spike density functions associated with noncanceled STOP trials and corresponding CONTROL trials never reached significance. These results suggest that, in our experiments, the STOP process did not influence the growth of the GO process.

\section{Responses of neurons with fixation-related activity}

In addition to neurons with saccade-related activity, we also collected data from a sample of neurons located within the rostrolateral portion of the SC (within the representation of very small saccades) and defined as providing an extraretinal fixation signal. Figure 7 shows the activity of a fixation-related neuron during countermanding trials. In the CONTROL trials, the activation of the neuron paused before and during the saccades. In the STOP trials, when the saccades were successfully canceled, the pause in activity was replaced by an increase in activity in response to the presentation of the STOP signal. In contrast to saccade-related neurons, fixation-related neurons must discharge more during canceled STOP trials than corresponding CONTROL trials to influence behavior. The discharge rate of this neuron during a 40 msec interval centered on the SSRT was significantly greater in canceled STOP trials $(96 \mathrm{~Hz})$ than corresponding CONTROL trials $(60 \mathrm{~Hz} ; t=-4.18$; $\mathrm{df}=129 ; p<0.0001)$. Furthermore, its neural estimate of saccade cancellation preceded (by $25 \mathrm{msec}$ ) the behavioral estimate obtained in these trials (129 msec). The activity of this neuron could therefore have been directly involved in countermanding the saccade that was being programmed, because the difference in activity occurred early enough within the SSRT.

For each fixation-related neuron, we calculated a ratio of the discharge rates during a $40 \mathrm{msec}$ interval centered on the SSRT in canceled STOP trials and corresponding CONTROL trials. Figure $8 \mathrm{~A}$ shows the distribution of these ratios calculated for each stop-signal delay (both target positions) in which sufficient trials were collected. The ratios ranged from 0.11 to 1.66 , with a mean $( \pm \mathrm{SE}$ ) of $0.6 \pm 0.04$, and $73 \%$ (46 of 63 ) of the ratios from all stop-signal delays available from all fixation-related neurons were significantly $<1$ ( $t$ test; $p<0.01$ ). Thus, a majority of fixation-related neurons displayed a discharge rate around the time of the SSRT that was significantly greater when saccades were canceled in STOP trials than when the saccades in CONTROL trials were produced but could have been canceled. Figure $8 \mathrm{~B}$ shows that similarly to saccade-related neurons, the neural estimates computed from fixation-related neurons preceded, on average by $13 \mathrm{msec}$, the behavioral estimates ( $\mathrm{SE}=2$; range, -38 

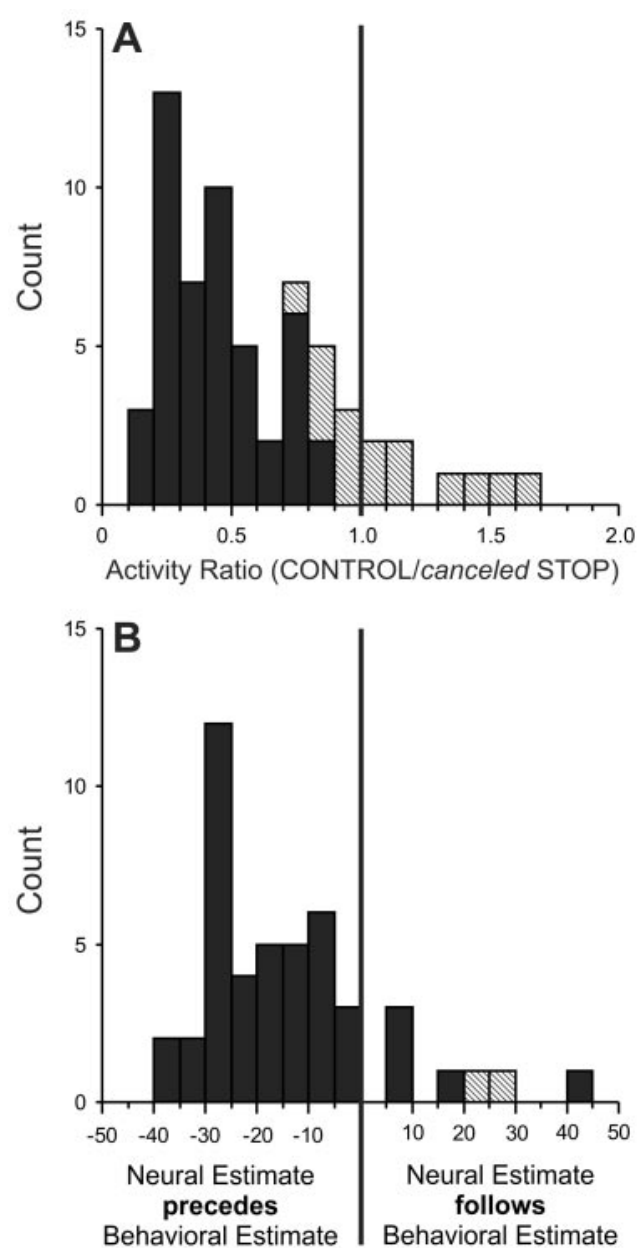

Figure 8. A, Distribution of the ratios of activity in the 40 msec interval centered on the time of the behavioral estimate of saccade cancellation in canceled STOP trials and corresponding CONTROL trials for the group of trials collected in each stop-signal delay in 10SC saccade-related neurons. Solid cells indicate the groups with activity ratios that were significantly smaller than unity. $B$, Distribution of the timing difference between the neural and behavioral estimates of saccade cancellation for the same fixation-related neurons.

to $44 ; n=46)$. In fact, the timing between the neural and behavioral estimates of saccade cancellation for these two groups of neurons was not significantly different from each other ( $t$ test; $p=0.19$ ), but both were significantly different from 0 ( $t$ test; $p<$ $0.0001)$.

\section{Discussion}

We used a countermanding saccade paradigm to gain new insights on how SC neurons are involved in saccade programming. We found that the activity of SC saccade-related neurons significantly dropped before saccades successfully countermanded in response to a visual stop signal presented at the fovea. Concomitantly, neurons with fixation-related activity increased their activity. Because the change in activity of these neurons occurred within the SSRT, leading the behavioral estimate of saccade cancellation by an interval exceeding the minimal amount of time needed for SC activity to influence eye movements, we conclude that the process of saccade cancellation fully involves the SC. The cancellation of the saccadic command can thus be viewed as the $a$ priori suppression of a saccadic command that was created centrally and not as the a posteriori suppression of a command that was already sent to the periphery. The additional observation that
SC saccade-related neurons discharged significantly less when saccades were countermanded instead of executed indicates that the SC neuronal activation may have to exceed a threshold level for a movement to be triggered. Taken as a whole, and together with the temporal coupling between the saccade-related burst of action potentials exhibited by these neurons and saccade initiation (Sparks, 1978), our results indicate that the SC exhibits the necessary discharge properties to be directly involved in regulating whether and when a saccade will be produced.

\section{Methodological issues}

Our conclusion depends critically on the quality of the behavioral estimate of the saccade cancellation process. We related changes in neuronal activity to the SSRT estimated in each recording session to account for variation of the animal's inhibition state between sessions. This approach could yield somewhat less reliable inhibition functions and regular distributions of reaction times, because the number of trials collected in each session was limited. Nonetheless, the estimate that we derived is similar to that reported in other monkeys (Hanes and Schall, 1995; Hanes et al., 1998), using the same analytical procedure. Furthermore, the mean difference between SC activity changes and the average behavioral estimates of saccade cancellation for each monkey was $11 \mathrm{msec}$ ( $\mathrm{SE}=1$; range, -37 to $35 ; n=92$ ), a value not significantly different from the $10 \mathrm{msec}$ obtained in the session-bysession analysis (paired $t$ test; $t=1.29 ; \mathrm{df}=90 ; p=0.20$ ). Our estimates were thus not systematically biased, at least not more than in other reports.

The second limitation of our analysis relates to possible errors in the neural estimate of saccade cancellation. The spike train filter that we used to compute spike density functions mimics the time course of postsynaptic potentials (1 msec rise time constant). The alternative and most common approach to convert spike train into density functions consists of convolving the spike trains with Gaussian filters (Waitzman et al., 1991). Analyzing our neural data with such filters (typically, $\sigma>3 \mathrm{msec}$ ) would have simply yielded neural estimates of saccade cancellation earlier than those we used. Thus, although not perfect, our analysis most likely did not overestimate the difference between the neural and behavioral estimates of each session. In addition, the fact that the ensemble of timing differences between neural and behavioral estimates showed some variability may be explained by non-covarying fluctuations in these two variables with respect to their means, which were compared.

\section{Inhibitory control of saccades}

The activity patterns that SC neurons exhibited during the countermanding saccade task had the necessary characteristics of neurons directly involved in the decision process regulating whether a saccade is to be made. The fact that the SC neural estimates of saccade cancellation almost consistently preceded the corresponding behavioral estimates is a remarkable result, given that the neural estimates from the other saccade executive center that has been investigated with the countermanding paradigm, the frontal eye field, have been shown to be coincident with saccade cancellation (Hanes et al., 1998). Although nearly all SC saccaderelated neurons $(98 \%)$ changed their activity within the SSRT, only $58 \%$ FEF saccade-related neurons did so. Moreover, this change in FEF activity led the behavioral estimate of saccade cancellation by an interval exceeding the FEF minimal efferent delay in no more than $48 \%$ of neurons, whereas this figure for SC neurons was $88 \%$. [The minimal amount of time needed for FEF activity to influence eye movements was estimated to be the sum 
of the SC minimal efferent delay (8 msec) (Miyashita and Hikosaka, 1996) and the average conduction time of the FEF connection to the SC (2 msec) (Segraves and Goldberg, 1987; Sommer and Wurtz, 2000).] We hypothesized that this FEF sample contained more than just FEF output neurons, and that these should change their activity appropriately to countermand saccades. Additional investigations are needed to resolve the timing discrepancy between SC and FEF neuronal modulation.

Within the SC, the saccade cancellation process was reflected to some extent in the decline of saccade-related activity and the rise of fixation-related activity. These activity patterns could have resulted from the direct activation of the fixation-related neurons in the SC by the foveal stop signal (Munoz and Wurtz, 1993), which would then have inhibited the saccade-related neurons via intracollicular connections (Munoz and Istvan, 1998). However, such a hypothesis appears inconsistent with the observed independence between the GO process, partially reflected in the discharge of saccade-related neurons, and the STOP process, partially reflected in the discharge of fixation-related neurons. The independence of these processes is central to the countermanding race model and our study, along with previous studies (Logan and Cowan, 1984; DeJong et al., 1990; Hanes and Schall, 1995; Hanes et al., 1998; Hanes and Carpenter, 1999), provides evidence consistent with this premise. However, it may be possible that interactions between SC fixation- and saccade-related signals occur during the very latest part of the race between the STOP and GO processes (e.g., to prevent the GO process from crossing the finish line and producing a saccade once the STOP process has won the race) (Hanes and Carpenter, 1999). Until then, the STOP process would act on the two types of SC signals separately. One candidate structure to provide such a dual influence onto the SC activity is the substantia nigra pars reticulata (SNpr), which has monosynaptic inhibitory projections to the SC (Hikosaka et al., 2000). SNpr neurons are known to exhibit either decreasing or increasing changes in activity associated with saccades made to visual stimuli (Handel and Glimcher, 1999, 2000; Sato and Hikosaka, 2002). We therefore surmise that the main component of the STOP process, reflected in SC activity, stems from a change in the selective inhibitory connections of SNpr pausers and bursters to the SC saccade- and fixation-related neurons, respectively.

In our view, the STOP process that countermands saccades involves a frontostriatal network, which has been associated with the suppression of reflexive saccades. Human patients with prefrontal cortex lesions have difficulty suppressing making saccades to a visual stimulus when instructed to make saccades away from the stimulus (Guitton et al., 1985; Pierrot-Deseilligny et al., 1991), and impairment in this anti-saccade task is associated with a selective failure in striatum activation (Raemaekers et al., 2002). Human imaging studies also suggest that response inhibition in Go/No-Go and stop tasks involves prefrontal areas (Casey et al., 1997; Bokura et al., 2001; Aron et al., 2003). The countermanding approach used in the FEF and SC must therefore be extended to the basal ganglia if we are to further our understanding of the inhibitory control of saccades.

\section{Central command for saccade production}

Before the presentation of a stop signal led to the successful cancellation of a planned saccade, SC saccade-related neurons discharged substantially, albeit always less than when saccades were made. This period of neuronal activation dissociable from saccade production could thus correspond to the controlled phase of saccade processing. Reduced activation associated with counter- manded saccades has also been observed in FEF (Hanes et al., 1998) as well as in the lateralized readiness potentials recorded when humans countermand manual responses (DeJong et al., 1990, 1995; Osman et al., 1992). These results provide additional evidence that the inhibitory control of saccades involves the suppression of a centrally generated saccadic command.

The difference between the maximum SC activity level associated with saccade cancellation and production is consistent with the hypothesis that a saccade is triggered only if neural activation surpasses a critical threshold, which defines the point of no return that separates the controlled and ballistic phases of processing. A likely neural substrate of the ballistic phase in saccade processing is the SC saccade-related burst in activity, which begins $\sim 20$ msec before a saccade (Sparks, 1978); FEF neurons have a $28 \mathrm{msec}$ lead (Hanes et al., 1995). Altogether, these data suggest that the point of no return occurs late during the GO process, thereby rendering the interruption of a planned movement very flexible (Osman et al., 1990).

\section{Conclusion}

Both saccade- and fixation-related neurons in the SC intermediate layers discharged differently when saccades were produced versus countermanded, and these changes in activity occurred within the SSRT. Furthermore, the difference between the time of occurrence of saccade cancellation estimated from the differential activity of these neurons and that estimated from the behavioral data simultaneously collected matched the SC minimal efferent delay. We consider these results as definitive evidence for the involvement of the SC in the inhibitory control of saccades.

\section{References}

Aizawa H, Wurtz RH (1998) Reversible inactivation of monkey superior colliculus. I. Curvature of saccadic trajectory. J Neurophysiol 78:2082-2096.

Aron AR, Fletcher PC, Bullmore ET, Sahakian BJ, Robbins TW (2003) Stopsignal inhibition disrupted by damage to right inferior frontal gyrus in humans. Nat Neurosci 6:115-116.

Band GPH, van der Molen MW, Logan GD (2003) Horse-race model simulations of the stop signal procedure. Acta Psychol (Amst) 112:105-142.

Becker W, Jürgens R (1979) An analysis of the saccadic system by means of double step stimuli. Vision Res 49:967-983.

Bokura H, Yamaguchi S, Kobayashi S (2001) Electrophysiological correlates for response inhibition in a Go/NoGo task. Clin Neurophysiol 112:2224-2232.

Carpenter RHS, Williams MLL (1995) Neural computation of log likelihood in control of saccadic eye movements. Nature 377:59-62.

Casey BJ, Trainor RJ, Orendi JL, Shubert AB, Nystrom LE, Giedd J, Castellanos FX, Haxby JV, Noll DC, Cohen JD, Forman SD, Dahl RE, Rapoport JL (1997) A developmental functional MRI study of prefrontal activation during performance of a Go-Nogo task. J Cognit Neurosci 9:835-847.

DeJong R, Coles MGH, Logan GD, Gratton G (1990) In search of the point of no return: the control of response processes. J Exp Psychol Hum Percept Perform 16:164-182.

DeJong R, Coles MGH, Logan GD (1995) Strategies and mechanisms in nonselective and selective inhibitory motor control. J Exp Psychol Hum Percept Perform 21:498-511.

Dorris MC, Munoz DP (1995) A neural correlate for the gap effect on saccadic reaction times in monkey. J Neurophysiol 73:2558-2562.

Dorris MC, Paré M, Munoz DP (1997) Neuronal activity in the monkey superior colliculus related to the initiation of saccadic eye movements. J Neurosci 17:8566-8579.

Edelman JA, Keller EL (1996) Activity of visuomotor burst neurons in the superior colliculus accompanying express saccades. J Neurophysiol 76:908-926.

Fuchs AF, Robinson DA (1966) A method for measuring horizontal and vertical eye movement chronically in the monkey. J Appl Physiol 21:1068-1070.

Guitton D, Buchtel HA, Douglas RM (1985) Frontal lobe lesions in man 
cause difficulties in suppressing reflexive glances and in generating goaldirected saccades. Exp Brain Res 58:455-472.

Handel A, Glimcher PW (1999) Quantitative analysis of substantia nigra pars reticulata activity during a visually guided saccade task. J Neurophysiol 82:3458-3475.

Handel A, Glimcher PW (2000) Contextual modulation of substantia nigra pars reticulata neurons. J Neurophysiol 83:3042-3048.

Hanes DP, Carpenter RHS (1999) Countermanding saccades in humans: evidence for a race-to-threshold process. Vision Res 39:2777-2791.

Hanes DP, Paré M (1998) Neural control of saccade production studied with the countermanding paradigm: superior colliculus. Soc Neurosci Abstr 24:418.

Hanes DP, Schall JD (1995) Countermanding saccades in macaque. Vis Neurosci 12:929-937.

Hanes DP, Schall JD (1996) Neural control of voluntary movement initiation. Science 274:427-430.

Hanes DP, Wurtz RH (2001) Interaction of the frontal eye field and superior colliculus for saccade generation. J Neurophysiol 85:804-815.

Hanes DP, Patterson Jr WF, Schall JD (1998) Role of frontal eye fields in countermanding saccades: visual, movement, and fixation activity. J Neurophysiol 79:817-834.

Hanes DP, Thompson KG, Schall JD (1995) Relationship of presaccadic activity in frontal eye field and supplementary eye field to saccade initiation in macaque: poisson spike train analysis. Exp Brain Res 103:85-96.

Hays AV, Richmond BJ, Optican LM (1982) A UNIX-based multiple process system for real-time data acquisition and control. Wescon Conf Proc 2:1-10.

Hikosaka O, Wurtz RH (1985) Modification of saccadic eye movements by GABA-related substances. I. Effect of muscimol and bicuculline in monkey superior colliculus. J Neurophysiol 53:266-291.

Hikosaka O, Wurtz RH (1986) Saccadic eye movements following injection of lidocaine into the superior colliculus. Exp Brain Res 61:531-539.

Hikosaka O, Takikawa Y, Kawagoe R (2000) Role of the basal ganglia in the control of purposive saccadic eye movements. Physiol Rev 80:953-978.

Lisberger SG, Fuchs AF, King WM, Evinger LC (1975) Effect of mean reaction time on saccadic responses to two-step stimuli with horizontal and vertical components. Vision Res 15:1021-1025.

Logan GD (1994) On the ability to inhibit thought and action: a users' guide to the stop signal paradigm. In: Inhibitory processes in attention, memory and language (Dagenbach D, Carr TH, eds), pp 189-239. San Diego: Academic.

Logan GD, Cowan WB (1984) On the ability to inhibit thought and action: a theory of an act of control. Psychol Rev 91:295-327.

Mason A, Nicoll A, Stratford K (1991) Synaptic transmission between individual pyramidal neurons of the rat visual cortex in vitro. J Neurosci 11:72-84.

Miyashita N, Hikosaka O (1996) Minimal synaptic delay in the saccadic output pathway of the superior colliculus studied in awake monkey. Exp Brain Res 112:187-196.

Munoz DP, Istvan PJ (1998) Lateral inhibitory interactions in the intermediate layers of the monkey superior colliculus. J Neurophysiol 79:1193-1209.

Munoz DP, Wurtz RH (1993) Fixation neurons in monkey superior colliculus I. Characteristics of neuron discharge. J Neurophysiol 70:559-575.

Munoz DP, Wurtz RH (1995) Saccade-related activity in monkey superior colliculus. I. Characteristics of burst and buildup neurons. J Neurophysiol 73:2313-2333.

Osman A, Kornblum S, Meyer DE (1986) The point of no return in choice reaction time: controlled and ballistic stages of response preparation. J Exp Psychol Hum Percept Perform 12:243-258.
Osman A, Kornblum S, Meyer DE (1990) Does motor programming necessitate response execution? J Exp Psychol Hum Percept Perform 16:183-198.

Osman A, Kornblum S, Meyer DE (1992) On the transmission of partial information: inferences from movement-related brain potentials. J Exp Psychol Hum Percept Perform 18:217-232.

Paré M (2003) Ballistic movement processing: saccade initiation is linked to an invariant trigger threshold in superior collicus. Soc Neurosci Abstr, in press.

Paré M, Wurtz RH (2001) Progression in neuronal processing for saccadic eye movements from parietal cortex area LIP to superior colliculus. J Neurophysiol 85:2545-2564.

Paré M, Crommelinck M, Guitton D (1994) Gaze shifts evoked by stimulation of the superior colliculus in the head-free cat conform to the motor map but also depend on stimulus strength and fixation activity. Exp Brain Res 101:123-139.

Pierrot-Deseilligny C, Rivaud S, Gaymard B, Agid Y (1991) Cortical control of reflexive visually-guided saccades. Brain 114:1473-1485.

Quaia C, Aizawa H, Optican LM, Wurtz RH (1998) Reversible inactivation of monkey superior colliculus. II. Maps of saccadic deficits. J Neurophysiol 79:2097-2110.

Raemaekers M, Jansma JM, Cahn W, van der Geest JN, van der Linden JA, Kahn RS, Ramsey NF (2002) Neuronal substrate of the saccadic inhibition deficit in schizophrenia investigated with 3-D event-related functional magnetic resonance imaging. Arch Gen Psychiatry 59:313-320.

Robinson DA (1972) Eye movement evoked by collicular stimulation in the alert monkey. Vision Res 12:1795-1808.

Sato M, Hikosaka O (2002) Role of primate substantia nigra pars reticulata in reward-oriented saccadic eye movement. J Neurosci 22:2363-2373.

Sayer RJ, Friedlander MJ, Redman SJ (1990) The time course and amplitude of EPSPs evoked at synapses between pairs of CA3/CA1 neurons in hippocampal slice. J Neurosci 10:826-836.

Schiller PH, True SD, Conway JL (1980) Deficits in eye movements following frontal eye-field and superior colliculus ablations. J Neurophysiol 44:1175-1189.

Schiller PH, Sandell JH, Maunsell JHR (1987) The effect of frontal eye field and superior colliculus lesions on saccadic latencies in the rhesus monkey. J Neurophysiol 57:1033-1049.

Segraves MA, Goldberg ME (1987) Functional properties of corticotectal neurons in the monkey's frontal eye field. J Neurophysiol 58:1387-1419.

Sommer MA, Wurtz RH (2000) Composition and topographic organization of signals sent from the frontal eye field to the superior colliculus. J Neurophysiol 83:1979-2001.

Sparks DL (1978) Functional properties of neurons in the monkey superior colliculus: coupling of neuronal activity and saccade onset. Brain Res 156:1-16.

Sparks DL, Lee C, Rohrer WH (1990) Population coding of the direction, amplitude, and velocity of saccadic eye movements by neurons in the superior colliculus. Cold Spring Harb Symp Quant Biol 55:805-811.

Stanford TR, Freedman EG, Sparks DL (1996) Site and parameters of microstimulation: evidence for independent effects on the properties of saccades evoked from the primate superior colliculus. J Neurophysiol 76:3360-3381.

Stuphorn V, Taylor TL, Schall JD (2000) Performance monitoring by the supplementary eye field. Nature 408:857-860.

Waitzman DM, Ma TP, Optican LM, Wurtz RH (1991) Superior colliculus neurons mediate the dynamic characteristics of saccades. J Neurophysiol 66:1716-1737. 